\title{
Multicuts in Planar and Bounded-Genus Graphs with Bounded Number of Terminals*
}

\author{
Éric Colin de Verdière ${ }^{\dagger}$
}

November 23, 2016

\begin{abstract}
Given an undirected, edge-weighted graph $G$ together with pairs of vertices, called pairs of terminals, the minimum multicut problem asks for a minimum-weight set of edges such that, after deleting these edges, the two terminals of each pair belong to different connected components of the graph. Relying on topological techniques, we provide a polynomial-time algorithm for this problem in the case where $G$ is embedded on a fixed surface of genus $g$ (e.g., when $G$ is planar) and has a fixed number $t$ of terminals. The running time is a polynomial of degree $O\left(\sqrt{g^{2}+g t}\right)$ in the input size.

In the planar case, our result corrects an error in an extended abstract by Bentz [Int. Workshop on Parameterized and Exact Computation, 109-119, 2012]. The minimum multicut problem is also a generalization of the multiway cut problem, a.k.a. multiterminal cut problem; even for this special case, no dedicated algorithm was known for graphs embedded on surfaces.
\end{abstract}

\section{Introduction}

The minimum cut problem is one of the most fundamental problems in combinatorial optimization (see Schrijver [34] for a fascinating historical account), originally introduced in relation to railway transshipment problems during the cold war 22. In this context, the railway network is modeled by a planar graph, each edge having a weight (its capacity), and the goal is to compute the minimum-weight set of edges that need to be removed to disconnect two given vertices of the network, the source and destination for a given commodity. While countless generalizations of this problem have been studied, we are interested here in two natural extensions:

1. What if there are several commodities, corresponding to different source and destination pairs? In other words, we are studying an instance of the minimum multicut problem: Given several pairs of source and destination vertices, how to find a minimum-weight set of edges to disconnect every destination vertex from its corresponding source?

2. What if the network is not planar, but includes a few tunnels and bridges? In other words, what happens if the graph is embedded, not in the plane, but on some fixed surface?

More formally, let $G=(V, E)$ be an undirected graph. Furthermore, let $T$ be a subset of vertices of $G$, called terminals, and let $R$ be a set of unordered pairs of vertices in $T$, called terminal pairs. A subset $E^{\prime}$ of $E$ is a multicut (with respect to $(T, R)$ ) if for every terminal pair $\left\{t_{1}, t_{2}\right\} \in R$, the vertices $t_{1}$ and $t_{2}$ are in different connected components of the graph $\left(V, E \backslash E^{\prime}\right)$. In the minimum multicut problem (also known as the minimum multiterminal cut problem), we assume in addition that $G$ is positively edge-weighted, and the goal is to find a multicut of minimum weight. We prove that this problem is polynomial-time solvable if $G$ is embedded on a fixed surface and the number $t$ of terminals is fixed. More precisely:

\footnotetext{
${ }^{*}$ Work supported by the French ANR Blanc project ANR-12-BS02-005 (RDAM).

${ }^{\dagger}$ CNRS, Laboratoire d'Informatique Gaspard Monge, Marne-la-Vallée, France. eric.colindeverdiere@u-pem.fr. Part of this work was done while the author was at CNRS, Département d'informatique, École normale supérieure, Paris, France.
} 
Theorem 1.1. Assume that $G$ is cellularly embedded on a surface (orientable or not) of Euler genus $g$. Then the minimum multicut problem can be solved in $(g+t)^{O(g+t)} n^{O\left(\sqrt{g^{2}+g t}\right)}$ time, where $t=|T|$ is the number of terminals and $n$ is the number of edges of $G$.

This is the first polynomial-time algorithm for this purpose, even when specialized to either the multiway cut problem (see below for details) or the planar version. Moreover, the $n^{O(\sqrt{t})}$ dependence in the number of terminals is unavoidable, assuming the Exponential Time Hypothesis [30], even in these two special cases.

\section{Comparison with Former Work}

Many instances of the minimum multicut problem are hard, even in very restricted cases. In particular, it is NP-hard in unweighted binary trees [7] and unweighted stars [20, Theorem 3.1], and even APX-hard in the latter case. In the case where the number of pairs of terminals is fixed and at least three, Dahlhaus et al. [14 have proved that the problem is APX-hard in general graphs; nonetheless, it becomes polynomial-time solvable for bounded-treewidth graphs, as proved by Bentz [2, Theorem 1], and fixed parameter tractable in the size of the solution for unweighted graphs [6]. The problem is even harder for directed graphs [2].

In the case where the graph is planar, the number of terminals is fixed, and they all lie on the outer face, Bentz [3] has given a polynomial-time algorithm for the minimum multicut problem. More recently [4], he has announced an algorithm for the same case, but removing the condition that the terminals lie on the outer face. Unfortunately, his proof has several flaws, leaving little hope for repair (see Appendix A). We give a faster algorithm that also works for graphs on arbitrary surfaces.

A special case that is somewhat more tractable is the multiway cut problem (a.k.a. the multiterminal cut problem); this is the case where the set of pairs of terminals comprises all possible pairs of distinct vertices in the set of terminals $T \subset V$. In the planar case, Dahlhaus et al. 14 have proved that it is still NP-hard, but Bateni et al. [1] have given a polynomial-time approximation scheme. Again in the planar case, the problem is also polynomial-time solvable if the number of terminals is fixed, as proved in the early 1990s [14,23. In stark contrast, the complexity of the multicut problem has remained open until now, although it is a very natural generalization of the multiway cut problem (the multicut problem is "dual" to the multicommodity flow problem, largely studied [33, Chapters 70-76]).

More recently, Klein and Marx have shown that the planar multiway cut problem can be solved in $2^{O(t)} n^{O(\sqrt{t})}$ time (where $n$ is the complexity of the graph) [26]; Marx has proved that the $n^{O(\sqrt{t})}$ dependence is the best one could hope for, assuming the Exponential Time Hypothesis (ETH) [30]. Our algorithm is more general since it deals with multicut, not multiway cut, and works on arbitrary surfaces; its running time, for fixed genus, is $t^{O(t)} n^{O(\sqrt{t})}$; while the $t^{O(t)}$ factor is slightly worse than the $2^{O(t)}$ of Klein and Marx, the second factor is the same, and optimal unless ETH is false. Since approximability in the planar case is very different for multicut and multiway cut, our result is surprising, since it shows that, as far as exact computation is concerned, both are (essentially) equally hard.

As a side note, it is easy to see that, to solve the multicut problem, it suffices to guess the partition of the terminals into connected components, and to solve a multiway cut problem in a higher genus surface. However, this reduction is not useful here, since we would get a worse dependence in $t$ if we were to prove the result for multiway cut on surfaces and use this reduction.

On the other hand, graph algorithms dedicated to graphs embedded on a fixed surface have flourished during the last decade. One reason is that many graphs arising in geometric settings are naturally embedded on a surface; another one is that the family of graphs embeddable on a fixed surface is closed under minor, and such studies can be seen as a first step towards efficient algorithms for minor-closed families. This line of research is also justified by the fact that testing whether a graph of complexity $n$ embeds on a surface of genus $g$ can be done efficiently in the complexity of the graph, namely, in $2^{O(g)} n$ time 31 . However, the history of flow and cut problems for graphs embedded on surfaces is rather short. Chambers et al. 9] have given an algorithm to compute a minimum cut in a graph embedded on an orientable surface of genus $g$ that runs in $g^{O(g)} n \log n$ time; a very different algorithm by Erickson and Nayyeri 17] runs in $2^{O(g)} n \log n$ time. Thus, the minimum cut problem is solvable in near-linear time on graphs embeddable on a fixed orientable surface. Algorithms 
are also available for computing global minimum cuts [16] and maximum flows 10 for graphs on surfaces. To our knowledge, we present here the first algorithm for the minimum multicut problem (or even the multiway cut problem) for surface-embedded graphs. The main tool for flows and cuts on surfaces 9, 10,16, 17 is homology, which is the appropriate algebraic formalism for dealing with graphs separating two given vertices, but it appears to be insufficient in the multicommodity case.

\section{Overview and Discussion of Proof Techniques}

The strategy for proving Theorem 1.1 is the following. In Section 3, we first show that a multicut corresponds, in a dual sense, to a graph drawn on $S$ that separates all pairs of terminals; such a graph will be called a multicut dual. Moreover, if the multicut is minimum, then this multicut dual is as short as possible, when distances on $S$ are measured using the cross-metric: namely, the sum of the weights of the edges of $G$ crossed by the multicut dual is minimum. The topological structure of the multicut dual can be described suitably after we cut the surface open into a disk with all terminals on its boundary (Section 4). We then show that this structure is constrained (Section 5), that we can enumerate its various possibilities (Section 6), and (roughly) that, for each of these topologies, we can compute a shortest multicut dual with that topology efficiently (Section 7).

At a high level, our approach follows a similar pattern to Klein and Marx [26, since they also rely on enumerating the various candidate topologies for the dual solution and find the optimum solution for each topology separately. This strategy is also present in Dahlhaus et al. [14] and, in a different context, in Chambers et al. [8], which was our initial source of inspiration. The details are, however, rather different.

Indeed, Klein and Marx [26] need a reduction to the biconnected case [26, Section 3], which is shortcut in our approach. Also, the structural properties that we develop for the minimum multicut problem are more involved than the known ones for the multiway cut problem; indeed, the solution, viewed in the dual graph, has less structure in the multicut problem than in the multiway cut problem: in the multiway cut case, it has as many faces as terminals, and thus many cycles, whereas for the minimum multicut problem, the possible topologies are more diverse (e.g., an optimal solution could be a single dual cycle).

Chambers et al. 8 have developed related techniques for computing a shortest splitting cycle, which have been subsequently reused for other topological and computational problems in planar or surface cases $9,18,19$. A key difference, however, is that we extend the method to work with graphs instead of paths or cycles, which makes the arguments more complicated. We need to encode precisely the locations of the vertices and edges of the multicut dual with respect to the cut graph; the cross-metric setting is very convenient for this purpose, since it avoids successive transformations of the input dual graph to mimic cutting along another graph, as done by Klein and Marx [26].

Moreover, our approach also relies on other techniques from computational topology, in particular, homology techniques developed for the single commodity minimum cut problem [9], homotopy techniques for shortest homotopic paths 13, 27, and treewidth techniques for the surface case 15.

Finally, we remark that we are not aware of any significantly simpler proof for the planar case: The construction of Section 4 can be simplified, and Lemma 7.2 is standard in that case, but the overall strategy would be the same.

\section{Preliminaries}

We recall here standard definitions on the topology of surfaces. For general background on topology, see for example Stillwell [35] or Hatcher [24]. For more specific background on the topology of surfaces in our context, see recent articles and surveys on the same topic 12,13 .

In this article, $S$ is a compact, connected surface without boundary; $g$ denotes its Euler genus. Thus, if $S$ is orientable, $g \geq 0$ is even, and $S$ is (homeomorphic to) a sphere with $g / 2$ handles attached; if $S$ is non-orientable, $S$ is a sphere with $g \geq 1$ disjoint disks replaced by Möbius strips.

We consider paths drawn on $S$. A path $p$ is a continuous map from $[0,1]$ to $S$; a loop is a path $p$ such that its two endpoints coincide: $p(0)=p(1)$. A path is simple if it is one-to-one (except, of course, that its endpoints $p(0)$ and $p(1)$ may coincide). We thus emphasize that, contrary to the 
standard terminology in graph theory, paths may self-intersect. A simple loop is two-sided if it has a neighborhood homeomorphic to an annulus; otherwise, it has a neighborhood homeomorphic to a Möbius strip (which implies that the surface is non-orientable), and is one-sided.

All the graphs considered in this article may have loops and multiple edges. A drawing of a graph $G$ on $S$ maps the vertices of $G$ to points on $S$ and the edges of $G$ to paths on $S$ whose endpoints are the images of the incident vertices. An embedding of $G$ is a "crossing-free" drawing: The images of the vertices are pairwise distinct, and the image of each edge is a simple path intersecting the image of no other vertex or edge, except possibly at its endpoints. We will mostly consider graph embeddings on $S$. A face of an embedding of $G$ on $S$ is a connected component of $S$ minus (the image of) $G$. A graph is cellularly embedded on $S$ if every face of the graph is an open disk. A cut graph of $S$ is a graph $G$ embedded on $S$ whose unique face is a disk. (In particular, a cut graph has no isolated vertex, unless it is reduced to a single vertex and $S$ is a sphere - this case does not occur in this paper.) Euler's formula states that, if $G$ is a graph cellularly embedded on $S$ with $v$ vertices, $e$ edges, and $f$ faces, then $v-e+f=2-g$.

Algorithmically, we can store graphs cellularly embedded on $S$ by their combinatorial map, which essentially records the cyclic ordering of the edges around each vertex; there are efficient data structures for this purpose [15,28].

\section{The Cross-Metric Setting}

In this section, we prove that a minimum multicut corresponds, in an appropriate sense, to a shortest graph satisfying certain properties.

We say that a graph $H$ embedded on $S$ is in general position with respect to our input graph $G$ if there are finitely many intersection points between $G$ and $H$, and each such point corresponds to a crossing between an edge of $G$ and an edge of $H$. The length of $H$ is the sum, over all crossing points between $G$ and $H$, of the weight of the corresponding edge of $G$. Note that an edge of $H$ can cross an edge of $G$ several times, and in such cases, the length of the edge of $H$ is computed by taking into account the multiplicity of intersections. In other words, $G$ is now seen as a graph that provides a discrete (or cross-metric) distance function on $S[13$. Algorithmically, we can store a graph $H$ in general position with respect to $G$ by recording the combinatorial map of the overlay of $G$ and $H$, obtained by adding vertices at each intersection point between $G$ and $H$ and subdividing edges of $G$ and $H$.

In the following, unless noted otherwise, all graphs drawn on $S$ will be in general position with respect to $G$. Moreover, whenever we consider distances between two points in $S$ (not lying on $G$ ) or lengths of paths in $S$, we implicitly consider them in the above cross-metric sense. In some clearly mentioned cases below (see Proposition 4.1), we will need to consider paths $p$ that are in general position with respect to $G$, except that some of their endpoints may lie on $G$. In such cases, the endpoints of $p$ are not taken into account for determining the length of $p$.

A multicut dual is a graph $C$ embedded on $S$ such that, for every pair $\left\{t_{1}, t_{2}\right\} \in R$, the vertices $t_{1}$ and $t_{2}$ are in different faces of $C$. As the terminology suggests, we have the following proposition, which will guide our approach.

Proposition 3.1. Let $C$ be a shortest multicut dual. Then the set $E^{\prime}$ of edges of $G$ crossed at least once by $C$ is a minimum multicut.

Proof. $E^{\prime}$ is a multicut, because any path in $G$ connecting a pair of terminals $\left\{t_{1}, t_{2}\right\} \in R$ must cross the multicut dual, and thus use one edge in $E^{\prime}$. Moreover, the weight of $E^{\prime}$ is at most the length of $C$.

To prove that $E^{\prime}$ is a multicut of minimum weight, it suffices to prove that, for every multicut $E^{\prime \prime}$ with weight $w$, there exists a multicut dual of length $w$. Consider the dual graph $G^{*}$ that has one vertex inside each face of $G$ and one edge $e^{*}$ crossing each edge $e$ of $G$. The subgraph of $G^{*}$ with edge set $\left\{e^{*} \mid e \in E^{\prime \prime}\right\}$ forms a multicut dual whose length is $w$.

As a side remark, it follows that the minimum multicut problem can be seen as a discrete version of the following topological problem: Given a metric surface $S$ with boundary, and a set $R$ of pairs of 
boundary components, compute a shortest graph on $S$ that separates every pair of boundaries in $R$. We are exactly solving this problem in the realm of cross-metric surfaces.

\section{Planarization}

Our algorithm starts by computing a cut graph $K$ of $S$ passing through all the terminals. We will also need some structural properties for $K$, detailed in the following proposition. If $S$ is the sphere (equivalently, if $G$ is planar), we could take for $K$ a shortest spanning tree of $T$ (with respect to the cross-metric setting), which can be obtained using a simple modification of any algorithm for computing minimum spanning trees in graphs. For the general case, we use a known construction, a so-called greedy system of arcs [8]. The following proposition summarizes the properties that we will use.

Proposition 4.1. In $O(n \log n+(g+t) n)$ time, we can compute a cut graph $K$ on $S$, whose $O(g+t)$ vertices contain $T$, and with $O(g+t)$ edges, each of which is a shortest path on $S$. Some vertices of $K$ may lie on $G$ (either on vertices or on edges).

Proof. We temporarily remove a small disk containing each terminal, the boundary of which crosses each edge of $G$ incident to that terminal exactly once, and crossing no other edge of $G$. This yields a surface with boundary $S^{\prime}$ that is naturally a cross-metric surface, because the intersection of the image of the graph $G$ with $S^{\prime}$ is also a graph $G^{\prime}$ embedded on $S^{\prime}$. On that surface, we compute a system of arcs [8. Section 5.1], namely, a set of disjoint, simple paths with endpoints on the boundary of $S^{\prime}$ that cut $S^{\prime}$ into a topological disk. Moreover, there are $O(g+t)$ paths (by Euler's formula), and the aforementioned construction guarantees that each path is roughly the "concatenation" of (at most) two shortest paths. More precisely, each path can be split into two shortest paths by inserting a degree-two vertex on an intersection point between $e$ and some edge of $G^{\prime}$. (It is actually a shortest system of arcs [11, Conclusion].)

Putting back the disks containing the terminals, and extending the arcs slightly inside these disks to the terminals, we obtain a cut graph $K$ satisfying the desired properties. The number of edges of $K$ is still $O(g+t)$, and the running time is $O(n \log n+(g+t) n)$ [8].

At a high level, the algorithm consists in (1) enumerating all possible "topologies" of the multicut dual with respect to $K,(2)$ for each of these possible topologies, computing a shortest multicut dual with that topology, and (3) returning the overall shortest multicut dual.

\section{$5 \quad$ Structural Properties of a Shortest Multicut Dual}

In this section, we prove some structural properties of a shortest multicut dual.

Consider all shortest multicut duals in general position with respect to $K \cup G$. Among all these, let $C_{0}$ be one that crosses $K$ a minimum number of times. We can, without loss of generality, assume that $C_{0}$ is inclusionwise minimal, in the sense that no edge can be removed from $C_{0}$ without violating the fact that it is a multicut dual. Of course, we can assume that $C_{0}$ has no isolated vertex. If $C_{0}$ has a degree-one vertex, we can "prune" it, by removing it together with its incident edge. If $C_{0}$ has a degree-two vertex that is not a loop, we can "dissolve" it, by removing it and identifying the two incident edges. Thus, we can assume that $C_{0}$ has minimum degree at least two, and that every degree-two vertex is the vertex of a connected component that is a loop.

\subsection{Crossing Bound}

We start with an easy consequence of Euler's formula.

Lemma 5.1. $C_{0}$ has $O(g+t)$ vertices and edges.

Proof. We first note that each face of $C_{0}$ contains at least one terminal: Otherwise, let $e$ be an edge incident to a face not containing a terminal; we could remove $e$ without violating the multicut dual 
(a)

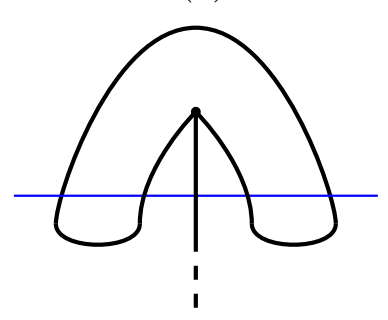

(b)

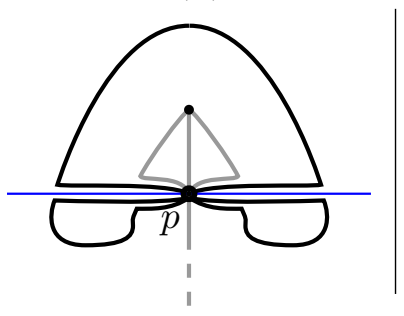

(c)

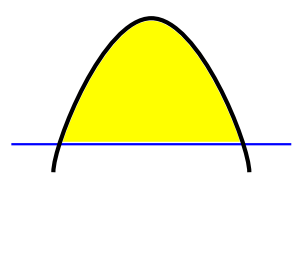

(d)

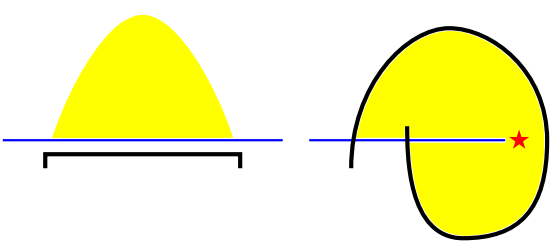

Figure 1: (a): The part of the multicut dual $C_{0}$ close to $e$ (depicted as a horizontal line). (b): its modified version $C_{0}^{\prime}$ obtained by pushing all crossings with $e$ to a single point $p$. The black lines are the loops in $L$, the grey ones are the other edges of $C_{0}^{\prime}$. (c): The configuration corresponding to a monogon. The disk is shaded. (d): The new configuration to replace (c). (e): This configuration is not a monogon, because of the presence of an obstacle (shown as a star).

property (because it would not change which pairs of terminals are separated), contradicting the minimality of $C_{0}$. Thus, $C_{0}$ has at most $t$ faces.

Let us first assume that every face of $C_{0}$ is a disk. If $C_{0}$ contains a degree-two vertex, this means that some connected component of $C_{0}$ is a loop $\ell$. Since each face of $C_{0}$ is a disk and $S$ is connected, the graph $C_{0}$ must be connected, so it equals $\ell$, and the statement of the lemma holds. So we can assume that $C_{0}$ has minimum degree at least three. Let $v, e$, and $f$ be the numbers of vertices, edges, and faces of $C_{0}$; we deduce that $3 v \leq 2 e$. Combining this with Euler's formula $v-e+f=2-g$ and the fact that $f \leq t$, we obtain that $e=O(g+t)$, and thus also $v=O(g+t)$, as desired.

If some faces of $C_{0}$ are not disks, we can iteratively extend $C_{0}$ by adding edges between existing vertices of $C_{0}$ so that no new face is created but each face is cut into a disk (see, e.g., Chambers et al. [8, proof of Lemma 2.1]; the proof in that article extends verbatim to non-orientable surfaces). Applying the reasoning of the previous paragraph to this new graph yields that it has $O(g+t)$ vertices and edges; this is also true for $C_{0}$.

The main structural property of $C_{0}$ is isolated in the following lemma:

Lemma 5.2. There are $O(g+t)$ crossings between $C_{0}$ and each edge of $K$.

As in algorithms for other problems using the same approach $8,9,18,19,26$, the proof of this lemma consists of an exchange argument: If $C_{0}$ crosses an edge of $K$ too many times, we can replace $C_{0}$ with a no longer multicut dual that crosses $K$ fewer times, contradicting the choice of $C_{0}$. The proof ultimately boils down to topological considerations. Let us also mention that the only property that we are using on the edges of $K$ is that they are disjoint shortest paths (except possibly at their endpoints).

Proof of Lemma 5.2. We focus on a specific edge $e$ of $K$ crossed by $C_{0}$, forgetting about the others. It is convenient to put an obstacle close to each of the two endpoints of $e$ (since $e$ is a shortest path, its endpoints are distinct). It is also convenient to temporarily look at the situation differently, by forgetting about $G$ and by modifying $C_{0}$ in the vicinity of $e$ by pushing all crossings of $C_{0}$ with $e$ to a single point $p$ on $e$ (Figure 1 $(\mathrm{a}, \mathrm{b}))$. This transforms $C_{0}$ into another graph $C_{0}^{\prime}$ that has $p$ as a new vertex. To prove the lemma, it suffices to prove that the degree of $p$ in $C_{0}^{\prime}$ is $O(g+t)$. Moreover, every non-loop edge of $C_{0}^{\prime}$ corresponds to one of the two endpoints of an edge of $C_{0}$, and there are $O(g+t)$ of these by Lemma 5.1. Hence, if we let $L$ be the one-vertex subgraph of $C_{0}^{\prime}$ made of the loops of $C_{0}^{\prime}$ based at $p$, it suffices to prove that the number of loops in $L$ is $O(g+t)$.

A monogon, resp. a bigon, is a face of $L$ that is, topologically, an open disk with one, resp. two, copies of $p$ on its boundary and containing in its interior no obstacle, no vertex of $C_{0}$, and no terminal. We first claim that no face of $L$ can be a monogon. Otherwise (Figure 1(c)), some edge $e^{\prime}$ of $C_{0}$ crosses $e$ twice consecutively, at points $x$ and $y$ say, such that the pieces of $e$ and $e^{\prime}$ between $x$ and $y$ bound a disk containing in its interior no obstacle, no vertex of $C_{0}$, and no terminal. Since the disk contains no obstacle, the boundary of the disk lies entirely on one side of $e$, as in Figure 1(c), and other cases such as the one shown in Figure 1(e) cannot occur. Since the disk contains no vertex of $C_{0}$, it 
(a)
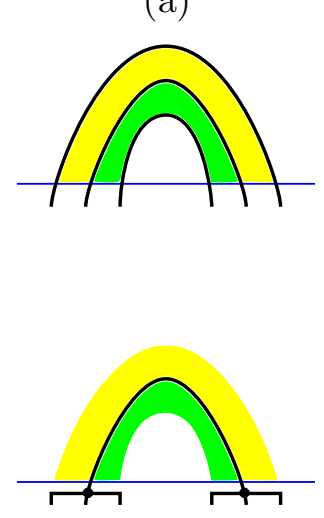

(b)

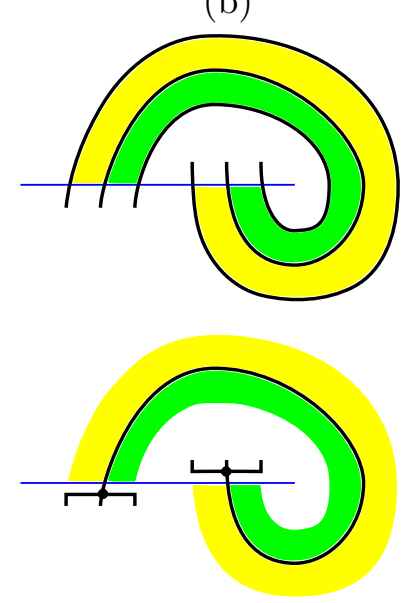

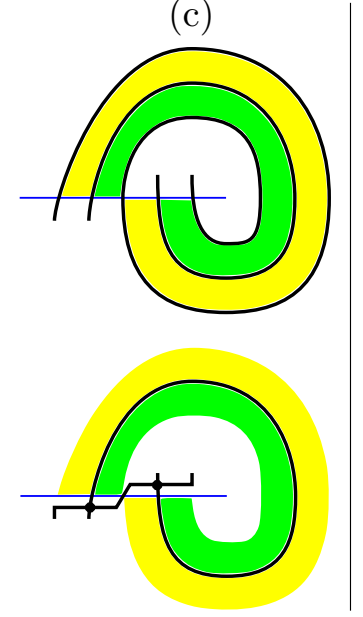

(d)

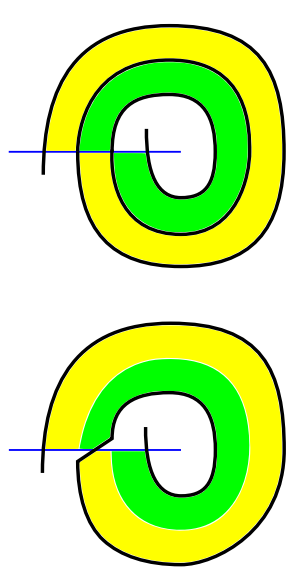

(e)
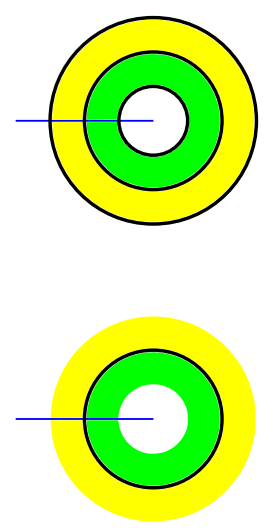

Figure 2: The exchange argument in the two-sided case. The horizontal segment represents edge $e$ of $K$. The strips are shaded; they represent disks with no terminal, no obstacle, and no piece of $C_{0}$ in their interior.

If the sides of the strips are all on the same side of $e$, there is a single case (a). Replacing the top configuration of $C_{0}$ with the bottom configuration (creating two new vertices) still yields a multicut dual (as all pairs of faces that were separated in the top configuration are still separated in the bottom configuration, except possibly for the strips, but these contain no terminal), which is no longer than the original (because $e$ is a shortest path) and has less crossings with $K$. This is a contradiction with the choice of $C_{0}$.

If the sides of the strips are on different sides of $e$, we need to distinguish according to four cases (b-e), depending on how the sides of the strips overlap. In all cases, the same argument shows that we could find a no longer multicut dual with fewer crossings with $K$, a contradiction. (We could also remark that case (e) is impossible because it involves closed curves in $C_{0}$ without vertex.)

contains no piece of $C_{0}$ in its interior. We can thus replace the piece of $e^{\prime}$ between $x$ and $y$ with a path that runs along $e$ (Figure 1(d)). This operation does not make $e^{\prime}$ longer, since $e$ is a shortest path; it removes the two intersection points with $e$ and does not introduce other crossings with $K$. Moreover, since the disk contains no terminal in its interior, the resulting graph is also a multicut dual. This contradiction with the choice of $C_{0}$ proves the claim.

We will prove below that no loop in $L$ can be incident to two bigons. Taking this fact for granted for now, whenever one face of $L$ is a bigon, we remove one of the two incident loops, and iterate until there is no bigon any more. The previous fact implies that these iterations remove at most half of the loops: If $L^{\prime}$ is the remaining set of loops, we have $|L| \leq 2\left|L^{\prime}\right|$. Furthermore, $L^{\prime}$ has no monogon or bigon. This latter fact, together with arguments based on Euler's formula, implies that the number of loops in $L^{\prime}$ is $O(g+t)$ [8, Lemma 2.1], because $S$ has Euler genus $g$, and the total number of obstacles, vertices of $C_{0}$, and terminals (which are the points that prevent a face that is a disk of degree one or two to be a monogon or bigon) is $O(g+t)$ (Lemma 5.1). (That article considers the orientable case only, but the lemma [8, Lemma 2.1] and its proof extend directly to the non-orientable case.) This implies that $|L|=O(g+t)$, which proves the lemma.

So there only remains to prove that no loop in $L$ can be incident to two bigons. Assume that such a loop exists. On the original surface $S$, this corresponds to two "strips" glued together, see Figure 2, top: Each strip is bounded by two pieces of $e$ and two pieces of edges of $C_{0}$, and these two strips share a common piece of edge of $C_{0}$. Since a bigon contains no obstacle, the sides of the strips contain none of the endpoints of $e$. Since the interiors of these strips contain no vertex of $C_{0}$, they contain no piece of $C_{0}$.

If $S$ is assumed to be orientable, there are five possible cases up to symmetry, see Figure 2, top: (a) is the case where each strip has its two sides on the same side of $e,(\mathrm{~b}-\mathrm{e})$ are the various cases where each strip has its two sides on opposite sides of $e$. In each case, we change $C_{0}$ by modifying some edges and possibly by adding vertices (see Figure 2, bottom). Since $e$ is a shortest path and the new pieces 
(a)
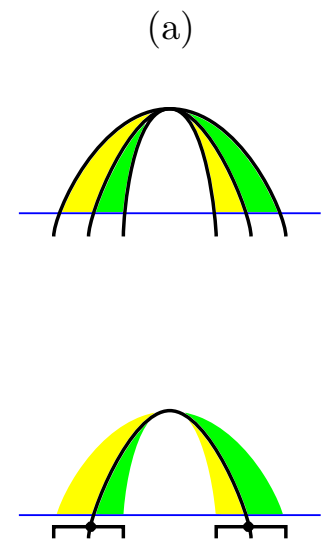

(b)

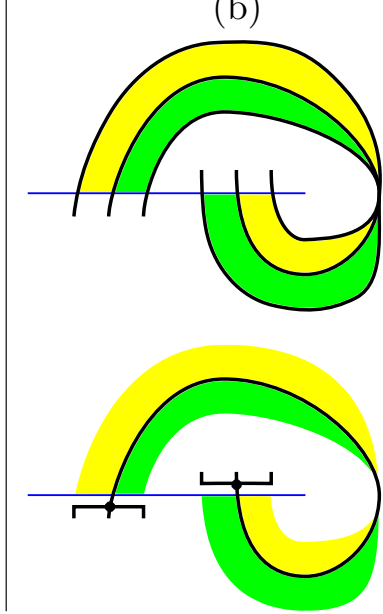

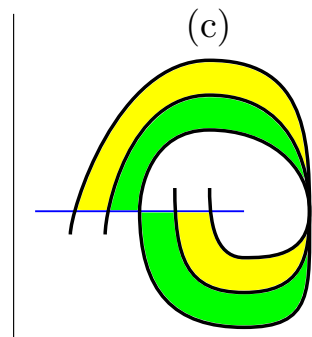

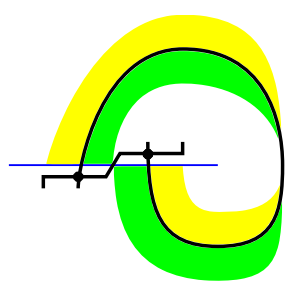

(d)

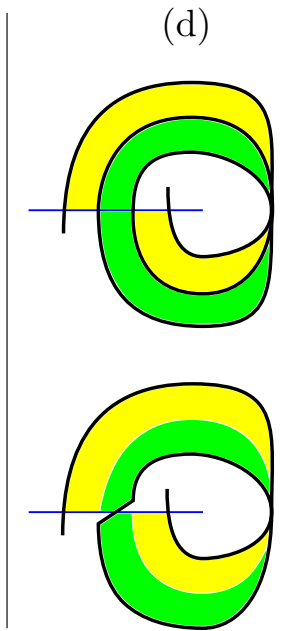

(e)

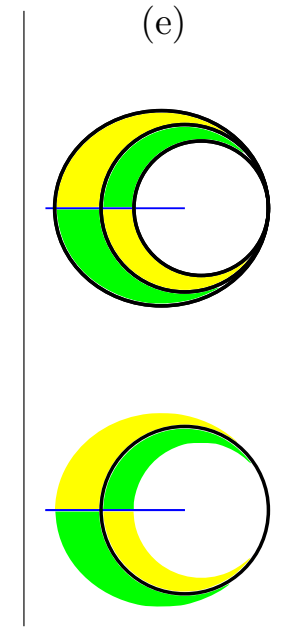

Figure 3: The exchange argument in the one-sided case. The argument is the same as in Figure 2 the sole difference is that the strips are drawn with a "twist".

"run along" $e$, one can check that the resulting graph is no longer than $C_{0}$; moreover, it crosses $K$ fewer times. Also, each replacement may split some faces of the original graph and attach each of the strips to some of the resulting faces, but pairs of terminals that were initially separated by $C_{0}$ are still separated by the new graph, which is thus also a multicut dual. This contradicts the choice of $C_{0}$.

If $S$ is non-orientable, there are five other cases, because the loops in $L$ may be one-sided. However, an entirely similar argument as in the previous paragraph (Figure 3 ) allows to conclude.

\subsection{Some Shortest Multicut Dual is Good}

We now give a more precise description of the intersection pattern between $C_{0}$ and $K$, using the properties proved in the previous section. Cutting the surface $S$ along $K$ yields a topological disk $D$. The boundary $\partial D$ of $D$ corresponds to copies of vertices and edges of $K$; there are $O(g+t)$ of these. The copies of the vertices of $K$ on $\partial D$ are called the corners of $D$, while the copies of the edges of $K$ are called the sides of $D$. The sides of $D$ can be reglued pairwise to obtain $S$.

Let $C$ be a graph on $S$ in general position with respect to $K \cup G$. In particular, $C_{0}$ is such a graph. Cutting $S$ along $K$ transforms the overlay of $K$ and $C$ into a graph $U$ drawn in $D$ (Figure 4 (a)): Each edge of that graph corresponds to a piece of an edge of $K$ or of $C$; each vertex of that graph corresponds to a vertex of $K$, a vertex of $C$, or a (four-valent) intersection point between an edge of $K$ and an edge of $C$. We denote by $\bar{C}$ the subgraph of $U$ made of the edges corresponding to pieces of edges of $C$ (thus, $\bar{C}$ lies in the interior of $D$ except possibly for some of its leaves), and by $\overline{\boldsymbol{K}}$ the subgraph of $U$ made of the edges corresponding to pieces of edges of $K$ (thus, the image of $\bar{K}$ is the boundary of $D$ ).

Definition 5.3. We say that $C$ is $\operatorname{good}$ if $\bar{C}$ is the disjoint union of (see Figure $4($ a)):

- trees with at least one vertex of degree at least two, with all their leaves on $\partial D$, and

- arcs, namely, edges with both endpoints on $\partial D$, on different sides of $\partial D$,

and, moreover:

- there are $O(g+t)$ intersection points between $C$ and each side of $\partial D$, and

- the total number of edges of the trees is $O(g+t)$.

Finally, Section 5 can be summarized as follows:

Proposition 5.4. Some shortest multicut dual is good. 
(a)

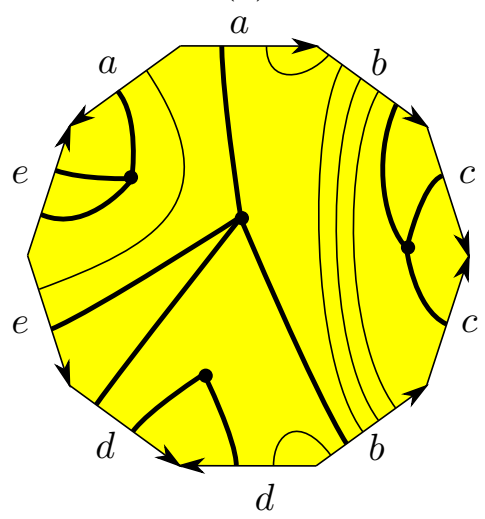

(b)

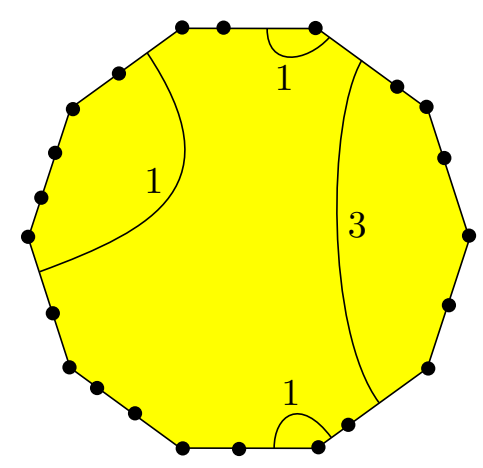

(c)

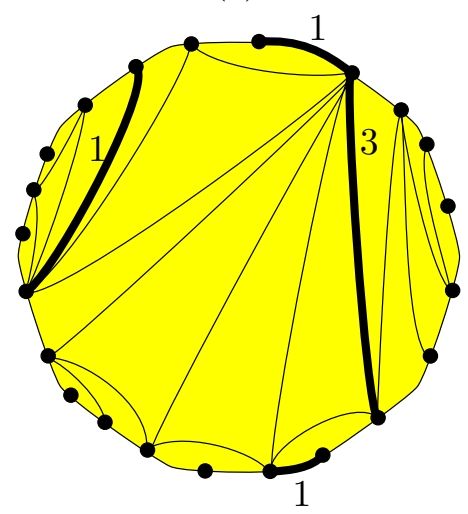

Figure 4: (a): The surface $S$, and a (good) graph $C$, viewed after cutting $S$ along the cut graph $K$, resulting in a disk $D$. The trees of $\bar{C}$ are in thick lines, the arcs being in thin lines. (b) and (c) are used to illustrate the proof of Proposition 6.2 (b) shows the disk $D$, with vertices on the boundary corresponding to the corners and the leaves of trees of $\bar{C}$; arcs connecting the same pair of edges of this polygon are represented by a single arc with an integer indicating their number; (c) represents the dual polygon (compared from (b), every vertex on the boundary is replaced with an edge, and every edge is replaced with a vertex); the arcs correspond to the internal or boundary edges of this dual polygon in thick lines; these edges can be augmented arbitrarily to a weighted triangulation of the dual polygon (the weights of the edges are zero unless noted otherwise).

Proof. We prove that $C_{0}$ is indeed good.

If some connected component of $\bar{C}_{0}$ contains a cycle, that cycle bounds a disk on $S$ containing no terminal (since the terminals are vertices of $K$ ), so we can remove any edge of that cycle without affecting the fact that $C_{0}$ is a multicut dual; this contradicts the choice of $C_{0}$. Therefore, $\bar{C}_{0}$ is a forest.

Since $C_{0}$ has no degree-zero or degree-one vertex, $\bar{C}_{0}$ has no isolated vertex, and any degree-one vertex of $\bar{C}_{0}$ lies on $\partial D$. Any component of $\bar{C}_{0}$ that is a single path must have its endpoints on different sides of $\bar{K}$; otherwise, we could take an innermost such path and "push it across" the side of $\bar{C}_{0}$ its endpoints lie in, using the same argument as in Figure 11 $(\mathrm{a}, \mathrm{b})$ : This would not make $C_{0}$ longer, and would decrease its number of crossings with $K$, contradicting the choice of $C_{0}$. This proves that $C_{0}$ satisfies the first two points of Definition 5.3. The third and fourth points trivially follow from Lemmas 5.2 and 5.1 , respectively.

\section{Enumerating Topologies}

Let $C$ be a good graph on $S$; recall that the union of $\bar{K}$ and $\bar{C}$ forms a connected planar graph $U$. The topology of $C$ is the data of the combinatorial map of $U$, where the outer face is distinguished, and the sides are paired. Intuitively, it describes combinatorially the relative positions of $C$ and $K$. More generally, a topology is the combinatorial map of a connected, planar graph with a distinguished outer face and a pairing of the sides (these are the subpaths of the outer cycle connecting consecutive degree-two vertices).

Lemma 6.1. Given a topology, we can determine whether it is the topology of a good graph that is a multicut dual in $O\left((g+t)^{2}\right)$ time.

Proof. First, we can assume that the topology has complexity $O\left((g+t)^{2}\right)$, since otherwise it is not the topology of a good graph. We first check that the outer boundary is a cycle. Then we glue the sides of the outer face according to the pairing. This results in the combinatorial map, on $S$, of the overlay of $C$ (corresponding to the interior edges of the topology) and $K$ (corresponding to the edges on the outer face - we can check that the combinatorial map indeed is the same as that of $K$ ). All of this takes $O\left((g+t)^{2}\right)$ time.

Deciding whether $C$ is good is easy in time linear in the size of the topology. Deciding whether $C$ is a multicut dual can also be done in that amount of time: We can determine which elements of $T$ fall 
into which face of $C$, since each terminal is a vertex of $K$, and whether each pair of terminals in $R$ lies in different faces of $C$.

This also implies that whether $C$ is a multicut dual or not is completely determined by its topology. Hence the following terminology: A topology is valid if it is the topology of a good graph that is a multicut dual. The result of this section can now be stated as follows.

Proposition 6.2. The number of valid topologies is $(g+t)^{O(g+t)}$; these topologies can be enumerated within the same time bound.

Proof of Proposition 6.2. It suffices to prove that there are $(g+t)^{O(g+t)}$ possible topologies for a good graph, and that we can enumerate them within the same time bound, because we can afterwards select the ones that correspond to multicut duals by Lemma 6.1.

We first focus on enumerating all the possibilities for the trees of $\bar{C}$, forgetting about the arcs. We know that the total number of edges of the forest is $O(g+t)$. Any such forest can be obtained by:

- starting with a tree $\tau$ with $O(g+t)$ edges where all internal vertices have degree three,

- contracting an arbitrary number of edges of that tree,

- removing an arbitrary number of edges of that tree.

Moreover, to specify exactly the topology for the trees of $\bar{C}$, we need to specify the cyclic ordering of the edges around each vertex of $\tau$, and to specify to which side of $\bar{K}$ each leaf of $\tau$ belongs.

There are $2^{O(g+t)}$ possibilities for the choice of the initial tree $\tau$ together with the cyclic ordering of the edges around each vertex of $\tau$ (this is essentially a Catalan number, counting the number of binary trees). Once this is determined, there are $(g+t)^{O(g+t)}$ possibilities to determine to which side of $\bar{K}$ each leaf of $\tau$ belongs (actually, $2^{O(g+t)}$, but this refinement is useless here). There remains to choose which of the $O(g+t)$ edges to contract or delete, and to specify to which of the $O(g+t)$ sides of $\bar{K}$ each leaf of $\tau$ belongs. To conclude, there are $(g+t)^{O(g+t)}$ possible choices for the combinatorial map for the union of the trees, which can be enumerated also in $(g+t)^{O(g+t)}$ time.

Given such a possibility for the trees, we bound the number of possibilities for choosing the arcs. The $O(g+t)$ leaves of the trees and the corners cut $\partial D$ into $O(g+t)$ intervals. The arcs connect distinct intervals (by definition of a good graph); moreover, the arcs are pairwise disjoint. Consider the dual polygon having one vertex per interval, such that each edge connects two consecutive intervals along $\bar{K}$ (Figure $4(\mathrm{~b}, \mathrm{c})$ ). Connect two vertices of the dual polygon by an internal edge of the dual polygon whenever there exists at least one arc connecting the corresponding pair of intervals. Since the arcs are non-crossing, the internal edges do not cross, and form a subset of a triangulation of the dual polygon. There are $2^{O(g+t)}$ possible triangulations, since the dual polygon has $O(g+t)$ vertices (this is again a Catalan number). To conclude, the number of possibilities for inserting the arcs, assuming the trees have already been chosen, is bounded from above by the number of weighted triangulations of the dual polygon, namely, triangulations where each (internal or boundary) edge bears a non-negative number that encodes the number of arcs of the corresponding type, which is thus $O(g+t)$. This number is $(g+t)^{O(g+t)}$. Moreover, enumerating all these possibilities can be done in the same amount of time.

\section{Dealing With Each Valid Topology}

The strategy for our algorithm is roughly as follows: For each valid topology, we compute a shortest graph $C$ with that topology; then we return the set of edges of $G$ crossed by the overall shortest graph $C$. Actually, we do not exactly compute an embedding of a shortest graph $C$; instead, we compute a shortest drawing (possibly with crossings) of the same graph, with some homotopy constraints; in particular, that drawing is no longer than the shortest embedding, and we prove that this also corresponds to a minimum multicut. To get an efficient algorithm, we use dynamic programming on small treewidth graphs 5 . The key proposition is the following. 
Proposition 7.1. Given a valid topology, we can compute, in $n^{O\left(\sqrt{g^{2}+g t}\right)}$ time, some multicut whose weight is at most the length of each multicut dual with that topology.

The following lemma allows to use treewidth techniques for surface-embedded graphs; it seems to be folklore, and is standard in the planar case.

Lemma 7.2. Given a graph $H$ with $p$ vertices, edges, and faces embedded on a surface of genus $g$, one can compute in $O(p \sqrt{p})$ time a path decomposition of the graph with width $O(\sqrt{g p})$.

Proof. We start by removing loops and multiple edges, in time $O(p)$. Note that the resulting graph (still denoted by $H$ ) is, as the original one, not necessarily cellularly embedded on the surface $S$ of genus $g$. However, the combinatorial map given by $H$ defines a cellular embedding of $H$ on the surface $S^{\prime}$ obtained by pasting a disk to each facial walk of $H$ (this idea is recurrent, e.g., in Mohar and Thomassen [32]). The genus $g^{\prime}$ of $S^{\prime}$ is at most that of $S$. (Indeed, $H$ could be augmented to a graph $H^{\prime}$ cellularly embedded on $S$ by adding edges, without increasing the number of vertices and faces; the cellular embeddings $H^{\prime}$ on $S$ and $H$ on $S^{\prime}$ have the same number of vertices and faces, but the first one has more edges; so $S$ has genus larger than $S^{\prime}$, by Euler's formula.)

Let $h$ be the number of vertices of $H$. Given the embedding of $H$ on $S^{\prime}$, one can compute in $O(h)$ time a small, balanced, planar separator $A$ for $H$ : a set of $O(\sqrt{g h})$ vertices whose removal leaves a planar graph with no connected component with more than $2 h / 3$ vertices [15. Theorem 5.1]. (That theorem states only the existence of the separator, but the proof immediately gives a linear-time algorithm, as mentioned in the remark right after its proof.)

We then compute a path decomposition of the planar graph $H^{\prime}:=H-A$ of width $O\left(\sqrt{h^{\prime}}\right)$ in $O\left(h^{\prime} \sqrt{h^{\prime}}\right)$ time, where $h^{\prime}$ is the number of vertices of $H^{\prime}$, using standard techniques. For example, one can compute a small, balanced, planar separator $B$ for $H^{\prime}$ made of at most $c_{1} \sqrt{h^{\prime}}$ vertices, for some constant $c_{1}[29]$; by induction, we can assume that each component of $H^{\prime}-B$ has a path decomposition of width at most $2 c_{2} h^{\prime} / 3$, for some constant $c_{2}$ to be chosen later; concatenating these paths arbitrarily and adding $B$ to all the nodes of the resulting path gives a path decomposition of $H^{\prime}$ of width at most $2 c_{2} h^{\prime} / 3+c_{1} \sqrt{h^{\prime}}$, which is at most $2 c_{2} h^{\prime}$ if $c_{2}$ is chosen large enough. By induction, it takes $O\left(h^{\prime} \sqrt{h^{\prime}}\right)$ time to compute this path decomposition explicitly. (Using more advanced techniques [21], and if one is willing to accept an implicit representation of the path decomposition, one can achieve the same result in linear time, but we do not care about this, since this will not improve the running time of the overall algorithm.)

Finally, adding $A$ to each node of the path decomposition of $H^{\prime}=H-A$ gives a path decomposition of $H$ of width $O(\sqrt{g p})$ in $O(p \sqrt{p})$ time.

Let $C$ be a good graph. The crossing sequence of an edge $e$ of $C$ (directed arbitrarily) is the ordered sequence of edges in $K$ crossed by $e$ when walking along $e$, together with the indication of the orientation of each crossing (more precisely, on which side of the edge of $K$ lies the part of $e$ before the crossing, and on which side lies the part of $e$ after the crossing). Given the topology of $C$, one can determine the crossing sequence of every edge of $C$. We say that a drawing $C^{\prime}$ of the (abstract) graph $C$ has the same topology as $C$ if each edge of $C^{\prime}$ has the same crossing sequence as the corresponding edge of $C$.

Lemma 7.3. Let $C^{\prime}$ be a drawing of a multicut dual $C$ with the same topology as $C$. Then, the set of edges of $G$ crossed by $C^{\prime}$ is a multicut.

Proof. Let $\left\{t_{1}, t_{2}\right\} \in R$ be a pair of terminals, and let $f$ be the face of $C$ containing $t_{1}$. The set of edges of $C$ that are incident exactly once to the face of $C$ containing $t_{1}$ forms an even subgraph $C_{1}$ of $C$, in which every vertex has even degree. Moreover, $C_{1}$ separates $t_{1}$ from $t_{2}$. Let $C_{1}^{\prime}$ be the drawing of the same subgraph in $C^{\prime}$. To prove our result, it suffices to prove that $C_{1}^{\prime}$ also separates $t_{1}$ from $t_{2}$, using the fact that the crossing sequences are the same in $C_{1}$ and $C_{1}^{\prime}$. Although intuitive, this fact is non-trivial, and its proof relies on two results involving homology. We will use these results as a black box; in particular, no knowledge of homology is required here.

We can assume that $C^{\prime}$ has a finite number of self-intersection points, each of which is a crossing. Therefore, $C_{1}^{\prime}$ can be seen as an even graph embedded on $S$ (by adding a new vertex at each crossing 
between two edges of $C_{1}^{\prime}$ ). Our lemma is implied by two results by Chambers et al. [9], reformulated here in our setting (that paper only considers orientable surfaces, but the two results we use extend immediately to non-orientable surfaces):

- if, for every edge $e$ of $K$, the even graphs $C_{1}$ and $C_{1}^{\prime}$ cross $e$ with the same parity, then they are homologous (over $\mathbb{Z} / 2 \mathbb{Z}$ ) [9, Lemma 3.4]. In our case, since the crossing sequences are equal, $C_{1}$ and $C_{1}^{\prime}$ are homologous;

- an even graph separates $t_{1}$ from $t_{2}$ if and only if it is homologous, on the surface $S \backslash\left\{t_{1}, t_{2}\right\}$, to a small circle around $t_{1}\left[9\right.$, Lemma 3.1]. Thus, since $C_{1}$ separates $t_{1}$ from $t_{2}$, it is also the case for $C_{1}^{\prime}$.

Proof of Proposition 7.1. Lemma 7.3 implies that it suffices to compute (the set of edges of $G$ crossed by) a shortest drawing with the given topology.

Let us first explain how to achieve this, assuming that the locations of the vertices are prescribed. Let $C$ be any multicut dual with that topology and these vertex locations; we do not know $C$, but know the crossing sequence of its edges (they are determined by the topology) and the vertex locations (they are prescribed). To achieve our goal, it suffices, for each edge $e$ of $C$, to compute a shortest path with the same crossing sequence as $e$ and the same endpoints. We remark that algorithms for computing a shortest homotopic path in $S$ minus the vertex set of $K$ precisely achieve this goal [13]: In short, we glue copies of the disk $D$ according to the specified crossing sequence, in such a way that edge $e$ "lifts" to that space $\hat{S}_{e}$; then we compute a shortest path in $\hat{S}_{e}$ connecting the same endpoints as that lift, and "project" back to $S$. (We can assign infinitesimal crossing weights to the edges of $K$ to ensure that the crossing sequences of $e$ and $e^{\prime}$ are the same.) The complexity of $D$ (with its internal structure defined by the edges of $G)$ is $O((g+t) n)$, and the crossing sequences have total length $O\left((g+t)^{2}\right)$, so the total complexity of the spaces $\hat{S}_{e}$ is $O\left((g+t)^{3} n\right)$. Since $\hat{S}_{e}$ is planar, and since shortest paths can be computed in linear time in planar graphs [25], this is also the complexity of computing (the set of edges of $G$ crossed by) a shortest graph drawing with a given topology and specified vertex locations.

To compute a shortest drawing with the given topology, over all choices of vertex locations, we can naïvely enumerate all possible locations of the $O(g+t)$ vertices. Note that it is only relevant to consider which face of the overlay of $G$ and $K$ each vertex belongs to, and there are $O((g+t) n)$ such faces. This yields an $n^{O(g+t)}$-time algorithm. To get a better running time, we use treewidth techniques, also used by Klein and Marx in the planar multiway cut case [26. The (abstract) graph $C$ defined by the specified topology has $O(g+t)$ vertices and is embedded on a surface with genus $g$. Lemma 7.2 gives us, in $(g+t)^{O(1)}$ time, a path decomposition of $C$ of width $O\left(\sqrt{g^{2}+g t}\right)$. We use standard dynamic programming on the path decomposition (rooted, e.g., at one of its endpoints). More precisely, at each node $N$ of the path, we have a table that indicates, for every choice of the locations of the vertices in $N$, the length of the shortest drawing of the subgraph of $C$ induced by the vertices in $N$ and its descendents, among those that respect the crossing sequence constraints. We can fill in the tables by a bottom-up traversal of the path decomposition. Since each node contains $O\left(\sqrt{g^{2}+g t}\right)$ vertices, the running time of the algorithm is $\left.n^{O\left(\sqrt{g^{2}+g t}\right.}\right)$.

We can now conclude the proof of Theorem 1.1 :

Proof of Theorem 1.1. We compute the cut graph $K$ in $O(n \log n+(g+t) n)$ time (Proposition 4.1), and enumerate all valid topologies in $(g+t)^{O(g+t)}$ time (Proposition 6.2). For each valid topology, we apply the algorithm of Proposition 7.1 in $n^{O\left(\sqrt{g^{2}+g t}\right)}$ time, and return a shortest multicut found. Therefore, the overall running time is $O\left((g+t)^{O(g+t)} n^{O\left(\sqrt{g^{2}+g t}\right)}\right.$. The correctness is easy: By Proposition 3.1 . it suffices to compute a multicut whose weight is at most the length of any multicut dual. By Proposition 5.4, some shortest multicut dual has a valid topology; when this topology is chosen in the course of the algorithm, Proposition 7.1 guarantees that we have computed a shortest multicut. 


\section{Acknowledgments}

Many thanks to Cédric Bentz for several helpful discussions on his manuscript [4]. Thanks also to Claire Mathieu for an inspiring discussion in the preliminary stage of this paper, to Arnaud de Mesmay for a useful remark, and to the anonymous referees for their detailed reports, one of them suggesting that an improvement might be possible with the treewidth technique of Klein and Marx [26].

\section{References}

[1] MohammadHossein Bateni, MohammadTaghi Hajiaghayi, Philip N. Klein, and Claire Mathieu. A polynomial-time approximation scheme for planar multiway cut. In Proceedings of the 23rd Annual ACM-SIAM Symposium on Discrete Algorithms (SODA), pages 639-655, 2012.

[2] Cédric Bentz. On the complexity of the multicut problem in bounded tree-width graphs and digraphs. Discrete Applied Mathematics, 156(10):1908-1917, 2008.

[3] Cédric Bentz. A simple algorithm for multicuts in planar graphs with outer terminals. Discrete Applied Mathematics, 157:1959-1964, 2009.

[4] Cédric Bentz. A polynomial-time algorithm for planar multicuts with few source-sink pairs. In International Workshop on Parameterized and Exact Computation, pages 109-119, 2012. Also in arXiv:1206.3999.

[5] Hans Bodlaender. Dynamic programming on graphs with bounded treewidth. In Proceedings of the 15th International Colloquium on Automata, Languages and Programming (ICALP), 1988.

[6] Nicolas Bousquet, Jean Daligault, and Stéphan Thomassé. Multicut is FPT. In Proceedings of the 43rd Annual ACM Symposium on Theory of Computing (STOC), pages 459-468, 2011.

[7] Gruia Călinescu, Cristina G. Fernandes, and Bruce Reed. Multicuts in unweighted graphs and digraphs with bounded degree and bounded tree-width. Journal of Algorithms, 48:333-359, 2003.

[8] Erin W. Chambers, Éric Colin de Verdière, Jeff Erickson, Francis Lazarus, and Kim Whittlesey. Splitting (complicated) surfaces is hard. Computational Geometry: Theory and Applications, 41(1-2):94-110, 2008.

[9] Erin W. Chambers, Jeff Erickson, and Amir Nayyeri. Minimum cuts and shortest homologous cycles. In Proceedings of the 25th Annual Symposium on Computational Geometry (SOCG), pages 377-385. ACM, 2009.

[10] Erin W. Chambers, Jeff Erickson, and Amir Nayyeri. Homology flows, cohomology cuts. SIAM Journal on Computing, 41(6):1605-1634, 2012.

[11] Éric Colin de Verdière. Shortest cut graph of a surface with prescribed vertex set. In Proceedings of the 18th European Symposium on Algorithms (ESA), part 2, number 6347 in Lecture Notes in Computer Science, pages 100-111, 2010.

[12] Éric Colin de Verdière. Topological algorithms for graphs on surfaces. Habilitation thesis, École normale supérieure, 2012. Available at http://monge.univ-mlv.fr/ colinde/pub/12hdr.pdf.

[13] Éric Colin de Verdière and Jeff Erickson. Tightening nonsimple paths and cycles on surfaces. SIAM Journal on Computing, 39(8):3784-3813, 2010.

[14] Elias Dahlhaus, David S. Johnson, Christos H. Papadimitriou, Paul D. Seymour, and Mihalis Yannakakis. The complexity of multiterminal cuts. SIAM Journal on Computing, 23(4):864-894, 1994.

[15] David Eppstein. Dynamic generators of topologically embedded graphs. In Proceedings of the 14th Annual ACM-SIAM Symposium on Discrete Algorithms (SODA), pages 599-608, 2003. 
[16] Jeff Erickson, Kyle Fox, and Amir Nayyeri. Global minimum cuts in surface embedded graphs. In Proceedings of the 23rd Annual ACM-SIAM Symposium on Discrete Algorithms (SODA), pages 1309-1318, 2012.

[17] Jeff Erickson and Amir Nayyeri. Minimum cuts and shortest non-separating cycles via homology covers. In Proceedings of the 22nd Annual ACM-SIAM Symposium on Discrete Algorithms (SODA), pages 1166-1176, 2011.

[18] Jeff Erickson and Amir Nayyeri. Shortest non-crossing walks in the plane. In Proceedings of the 22nd Annual ACM-SIAM Symposium on Discrete Algorithms (SODA), pages 297-308, 2011.

[19] Kyle Fox. Shortest non-trivial cycles in directed and undirected surface graphs. In Proceedings of the 24th Annual ACM-SIAM Symposium on Discrete Algorithms (SODA), pages 352-364, 2013.

[20] Naveen Garg, Vijay V. Vazirani, and Mihalis Yannakakis. Primal-dual approximation algorithms for integral flow and multicut in trees. Algorithmica, 18:3-20, 1997.

[21] Michael T. Goodrich. Planar separators and parallel polygon triangulation. Journal of Computer and System Sciences, 51(3):374-389, 1995.

[22] T. E. Harris and F. S. Ross. Fundamentals of a method for evaluating rail net capacities. Technical Report Research Memorandum RM-1573, The RAND Corporation, Santa Monica, California, 1955 .

[23] David Hartvigsen. The planar multiterminal cut problem. Discrete Applied Mathematics, 85:203$222,1998$.

[24] Allen Hatcher. Algebraic topology. Cambridge University Press, 2002. Available at http: //www.math. cornell.edu/ hatcher/.

[25] Monika R. Henzinger, Philip Klein, Satish Rao, and Sairam Subramanian. Faster shortest-path algorithms for planar graphs. Journal of Computer and System Sciences, 55(1, part 1):3-23, 1997.

[26] Philip N. Klein and Dániel Marx. Solving Planar $k$-Terminal CUT in $O\left(n^{c \sqrt{k}}\right)$ time. In Proceedings of the 39th International Colloquium on Automata, Languages and Programming (ICALP) volume 1, pages 569-580, 2012.

[27] Martin Kutz. Computing shortest non-trivial cycles on orientable surfaces of bounded genus in almost linear time. In Proceedings of the 22nd Annual Symposium on Computational Geometry (SOCG), pages 430-438. ACM, 2006.

[28] Sóstenes Lins. Graph-encoded maps. Journal of Combinatorial Theory, Series B, 32:171-181, 1982.

[29] Richard J. Lipton and Robert Endre Tarjan. A separator theorem for planar graphs. SIAM Journal on Applied Mathematics, 36(2):177-189, 1979.

[30] Dániel Marx. A tight lower bound for planar multiway cut with fixed number of terminals. In Proceedings of the 39th International Colloquium on Automata, Languages and Programming (ICALP) volume 1, pages 677-688, 2012.

[31] Bojan Mohar. A linear time algorithm for embedding graphs in an arbitrary surface. SIAM Journal on Discrete Mathematics, 12(1):6-26, 1999.

[32] Bojan Mohar and Carsten Thomassen. Graphs on surfaces. Johns Hopkins Studies in the Mathematical Sciences. Johns Hopkins University Press, 2001.

[33] Alexander Schrijver. Combinatorial optimization. Polyhedra and efficiency, volume 24 of Algorithms and Combinatorics. Springer-Verlag, Berlin, 2003. 
[34] Alexander Schrijver. On the history of combinatorial optimization (till 1960). In Handbook of Discrete Optimization, pages 1-68. Elsevier, 2005.

[35] John Stillwell. Classical topology and combinatorial group theory. Springer-Verlag, New York, second edition, 1993.

\section{A Problems with Bentz' Approach in the Planar Case}

Here, we show several flaws in Bentz' approach [4] for the planar version of the problem, which leaves little hope for repair. Bentz starts by guessing the clusters, namely, the partition of the terminals induced by the faces of the optimal multicut dual, and enumerates all possibilities for the locations of the vertices of the multicut dual that have degree at least three. (He does so in the dual graph, while we do it in the cross-metric setting to keep track of the crossings between the multicut dual and the cut graph, but this is the same idea.) Then, his strategy is to consider all the possible homotopy classes of the closed walks $C_{i}$ that bound a face of the multicut dual containing the terminals of a given cluster $i$. Unfortunately, it is dubious that this strategy could work; at least, the enumeration of the homotopy classes has to be substantially more complicated than described in his article, and one would need to consider the homotopy classes of the edges of the multicut dual, not only of the closed walks $C_{i}$. In more detail:

- In Lemma 3, it is claimed that two cycles are homotopic in the plane minus a set of terminals if and only if they enclose the same terminals in their interior. This is not the case (see Figure 5). In particular, as soon as there is more than two terminals, the number of homotopy classes is infinite;

- Lemma 5 claims the following: Assume that $C_{i}$ passes through vertices $\omega_{1}, \ldots, \omega_{q_{i}}$ of the multicut dual. Then we still have a multicut dual if we replace $C_{i}$ with any cycle $C_{i}^{\prime}$ going through $\omega_{1}, \ldots, \omega_{q_{i}}$ that encloses the same set of terminals as $C_{i}$. This is not the case, as Figure 6 demonstrates. (Actually, Lemma 5 makes a slightly stronger claim, which is also contradicted by that figure.) For the same reason, Corollary 1 does not hold.

These counterexamples show that it is not sufficient to determine whether cycles are valid (can be part of a multicut dual) based only on which terminals they enclose (although this would indeed be very nice, since the number of such possibilities is $\left.n^{O(t)}\right)$. We really need to consider the homotopy classes of the paths, and to bound their number. This is exactly the purpose of Lemma 5.2 in our technique.
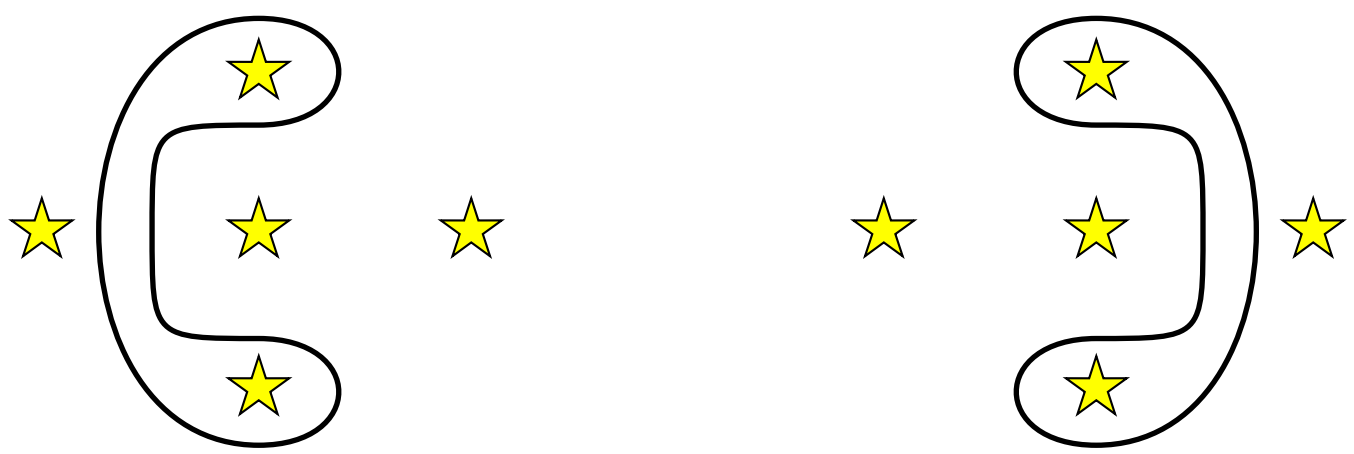

Figure 5: Two non-homotopic cycles enclosing the same set of terminals. 

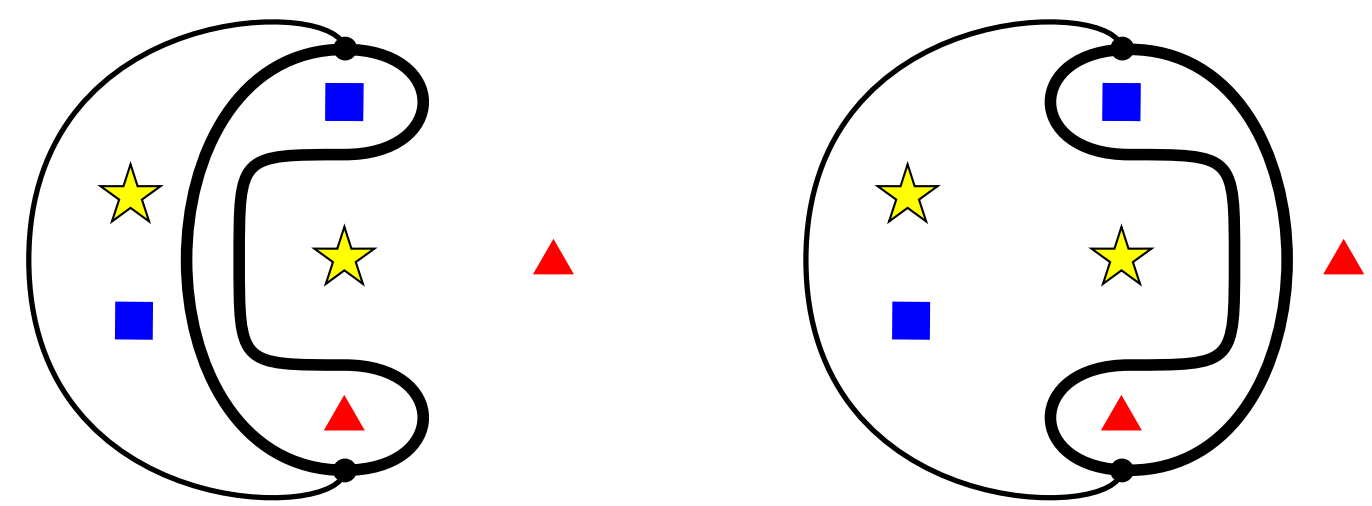

Figure 6: In a multicut dual, replacing a cycle (in thick lines) with another cycle passing through the same vertices and enclosing the same terminals does not always give a multicut dual. 